Meta

Journal des tradlucteurs

Translators' Journal

\title{
Il n'y a pas de suffixe-ateur en français, voyons! (III)
}

\section{Antoine Di-Lillo}

Volume 28, numéro 2, juin 1983

URI : https://id.erudit.org/iderudit/002567ar

DOI : https://doi.org/10.7202/002567ar

Aller au sommaire du numéro

Éditeur(s)

Les Presses de l'Université de Montréal

ISSN

0026-0452 (imprimé)

1492-1421 (numérique)

Découvrir la revue

Citer cet article

Di-Lillo, A. (1983). Il n’y a pas de suffixe-ateur en français, voyons! (III). Meta, 28(2), 157-166. https://doi.org/10.7202/002567ar d'utilisation que vous pouvez consulter en ligne.

https://apropos.erudit.org/fr/usagers/politique-dutilisation/ 


\section{ÉTUDES \\ TERMINOLOGIQUES ET LINGUISTIQUES}

\section{IL N'Y A PAS DE SUFFIXE-ATEUR EN FRANÇAIS, VOYONS! (III)}

\section{PRÉAMBULE}

La première et la seconde partie de cet article ont été consacrées à démontrer que les formes en -eur ne forment pas un ensemble monolithique mais, au contraire, qu'elles se divisent en deux classes bien distinctes du point de vue de leur dérivation: L'une, que nous avons appelée X-eur, dont les mots finissent directement en -eur (tels assur-eur ou chroniqu-eur), l'autre, les X-t-eur, dont eur est précédé, au moins, de la consonne -t-, cette consonne ne pouvant a priori faire partie du radical (tels administr-at-eur, contribu-t-eur, répét-it-eur). Nous avons en effet vu que les X-eur dérivent pour la plupart de bases verbales et, moins fréquemment, de base nominales monomorphémiques; quant au $\mathrm{X}$-t-eur, la seule analyse qui rende compte adéquatement de leur formation est celle qui les fait dériver du mot en -t-ion apparenté.

Nous rappelons que nous avions quatre arguments principaux en faveur de notre analyse : 1) Les X-t-eur ont pratiquement toujours un corrélat X-t-ion. 2) Il y a une correspondance morphologique pratiquement absolue entre $X$-t-eur et $\mathrm{X}$-t-ion. 3) Il y a des paires attestées du genre receveur $\sim$ récepteur que seule notre analyse peut expliquer. 4) La dérivation des X-t-eur sans corrélat verbal ne peut être expliquée que par une analyse qui part de la forme nominale en -t-ion et non du verbe.

Notre intention, dans cette dernière partie de l'article, sera de montrer comment notre hypothèse peut s'intégrer dans une théorie de la morphologie assez récente, celle de Lieber (1980); à condition toutefois de modifier ce modèle de façon à le nantir de règles de formation de radicaux qui sont de toutes façons indépendamment motivées pour le français comme nous le verrons, règles qui pourraient avantageusement remplacer les règles de troncation d'Aronoff. Nous allons auparavant présenter l'analyse que fait Zwanenburg (1980) des formes en -t-eur (entre autres); cet auteur se sert des résultats de son analyse pour étayer le modèle morphologique qu'il propose. Zwanenburg s'attaque en fait à l'analyse d'Aronoff qui veut rendre compte de la dérivation des formes en -or de l'anglais à partir des radicaux verbaux contenus dans les mots en -t-ion; et établissant un parallèle avec le français, Zwanenburg veut montrer que cette analyse ne serait pas adéquate pour rendre compte des dérivés en t-eur notamment. Bien que notre traitement soit tout à fait différent de celui d'Aronoff, puisque le sien part du verbe et le nôtre du nom, les contre-arguments 
qu'utilise Zwanenburg pourraient s'adresser à nous. C'est pour cette raison qu'après avoir brièvement esquissé le modèle de Zwanenburg nous réfuterons ses contre-arguments. Nous passerons ensuite à la formalisation de nos règles dans un cadre théorique précis.

\subsection{L'analyse de Zwanenburg (1980) ${ }^{1}$}

Les vues de Zwanenburg diffèrent de celles d'Aronoff (1976) essentiellement sur deux points: Aronoff postule que les règles de formation de mots n'opèrent que sur des mots de catégories lexicales majeures (noms, adjectifs, verbes et adverbes), alors que Zwanenburg affirme que les règles de formation de mots doivent pouvoir opérer également sur des morphèmes liés, c'est-à-dire n'apparaissant jamais à l'état isolé. Aronoff propose des règles de formation de mots qui opèrent de gauche à droite pour la formation de mots nouveaux et de droite à gauche pour analyser les mots existants, tandis que Zwanenburg limite le fonctionnement de ses règles de gauche à droite.

Pour démontrer que les règles de formation de mots doivent pouvoir opérer à partir de morphèmes liés, Zwanenburg invoque la formation du mot anglais transmote qu'Aronoff décrit, à tort selon lui, comme le soudage arbitraire des extrémités de deux mots. Zwanenburg refuse de voir en transmote le produit d'un agencement arbitraire, mais la combinaison de deux morphèmes qu'on trouve dans d'autres mots : trans- et mote. Son raisonnement est donc que, puisqu'on a des mots formés à partir de morphèmes liés, il faut prévoir des règles capables d'en rendre compte.

Pour ce qui est du sens des règles, écoutons Zwanenburg (1980b) :

Qu'on suive maintenant Jackendoff ou Aronoff en ce qui concerne l'opération de droite à gauche des règles de formation de mots, dans les deux cas on rencontre des difficultés très grandes, sinon insurmontables. C'est que, dans la grande majorité des cas, les règles de formation de mots sont soumises à des conditions morphologiques et phonologiques positives et négatives. C'est le cas par exemple des noms d'action français en -tion : ceux dérivés d'un verbe en -er se termineront en -ation, ceux dérivés d'un verbe en -ir à radical allongé prendront -ition et ceux qui remontent à un autre verbe auront -tion. Une exception importante à cette régularité est constituée par les verbes en -er qui sont à interpréter historiquement parlant comme des formations régressives, tels contracter/contraction par opposition à abstraire/abstraction ${ }^{2}$ (p. 4).

Zwanenburg mentionne que le principal argument que fournit Jackendoff en faveur du fonctionnement de droite à gauche des règles est qu'il n'est pas souhaitable d'entrer au lexique des mots inexistants du type *perdite ou

1. Nous tenons à exprimer notre reconnaissance à cet auteur qui nous a fait parvenir personnellement, dans les plus brefs délais, ses deux articles avant leur publication.

2. Il serait intéressant et pratique qu'on puisse aussi simplement rendre compte des formes en tion; si cela était le cas on pourrait rendre compte aussi simplement de la distribution des variances de -eur; or, nous avons vu un peu plus haut que la distribution n'était prévisible d'aucune manière à partir du radical verbal. Nous avons d'autre part montré dans Di-Lillo (1981) que la distribution des variantes du suffixe -ion (-ion, -ation, -ition, etc.) ne suivait aucune règle ayant comme base le radical verbal; ces formes lexicalisées depuis longtemps ne peuvent être décrites dans la grande majorité que par des règles de redondance; seule la variante -ation connaît une certaine productivité à partir de verbes de type $\mathrm{X}$-iser et peut donner lieu à une règle productive de formation de mots. 
* retribute (pour rendre compte de perdition ou de retribution) qui devront être marqués [-insertion lexicale] à cause de la puissance beaucoup trop grande d'un tel dispositif. Zwanenburg avance qu'il est possible d'éviter ce problème puisque, tous les mots existants étant spécifiés dans le lexique avec leur structure morphologique, tous les morphèmes ne se rencontrant pas à l'état isolé sont spécifiés dans les entrées lexicales des mots complexes où ils se rencontrent. Or, ditil, en permettant aux règles de formation de mots d'opérer sur ces morphèmes, de même que sur la sortie des règles de formation de mots et sur les mots existants, on évite ainsi à la fois des entrées marquées [-insertion lexicale] et les règles fonctionnant en sens inverse. Il montre que, par exemple, pour deduction, qui correspond à deduce, la règle opère sur un morphème verbal se rencontrant ailleurs dans le lexique à l'état isolé, alors que pour retribution, par exemple, qui n'a pas de verbe, la règle opère sur un morphème verbal auquel ne: correspond pas une entrée indépendante.

Zwanenburg est d'avis que les règles de troncation, qui de toute façon n'ont pas grand place dans son modèle, ne jouent pas le rôle important qu'Aronoff veut leur faire jouer en anglais. Il tente de démontrer que l'analyse par troncation des adjectifs en -ive, -or et -ory de l'anglais, à partir de radicaux verbaux qu'on retrouve dans les mots en -tion, qu'Aronoff propose, n'est pas justifiée. Il reproche à Aronoff de fonder son analyse sur le simple fait qu'il y a plus de mots en -tion que de mots en -ive, -or et -ory.

En outre, nous dit Zwanenburg (1980b) : «(cela) n'empêche pas littéralement Aronoff d'intégrer les mots en -ive, etc. auxquels ne correspond pas un nom d'action en -ion dans sa description : pour ce faire il aura recours à une règle d'analyse» (p. 9). Par règle d'analyse, l'auteur entend des règles opérant «de droite à gauche, en prenant le mot dérivé comme point de départ et en décomposant ce mot dans la mesure du possible en base plus affixe(s)» (p. 1).

Zwanenburg veut aussi démontrer qu'il serait injustifié d'appliquer l'analyse d'Aronoff au français, c'est-à-dire dériver les mots en -teur, -tif et -toire à partir des radicaux verbaux qu'on extrairait, par troncation du suffixe, du mot en -tion. Il va sans dire que Zwanenburg désire rendre compte de toutes ces formes par des règles de formation de mots opérant à la fois à partir de radicaux verbaux et de morphèmes verbaux. Voici d'ailleurs le schéma de règles proposé par cet auteur pour rendre compte, selon lui, des ressemblances de forme, de sens et de distribution de ces dérivés ${ }^{3}$ :

(42) $\left.\left.[\mathrm{X}] \mathrm{V} \rightarrow\left[[\mathrm{X}] \mathrm{v} t \quad\left\{\begin{array}{l}\text { ion } \\ \text { eur } \\ \text { if } \\ \text { oire }\end{array}\right]\right]_{\mathrm{N}}\right\} \mathrm{A}\right\} \begin{aligned} & \text { 'action de V' } \\ & \text { 'qui V' } \\ & \text { 'qui (sert à) V' } \\ & \text { 'qui (sert à) V' }\end{aligned}$

Il s'impose ici de rappeler que l'analyse que Zwanenburg tente de réfuter est celle d'Aronoff, et que cette analyse diffère de la nôtre en ce sens que nous postulons que c'est le mot en -tion qui sert directement de base au X-t-eur et non le radical verbal à la base du mot en -tion; cela implique que pour nous

3. L'auteur ne nous indique pas comment rendre compte du fait que le radical finit en $/ t /$ devant -eur mais en /s/devant -ion. 
le radical verbal n'est pas pertinent, d'autant plus qu'il peut ne pas exister. Cela dit, les arguments dont se sert Zwanenburg pour réfuter toute analyse à la Aronoff portent sur le parallélisme entre X-t-eur et X-t-ion. Or, nous avons vu que ce parallélisme est un de nos arguments également (mais non le plus important). C'est donc principalement pour cela que nous aimerions répondre à cet auteur. Voici ce que nous dit Zwanenburg (1980a) et qui constitue tous ses arguments :

(i) On trouve pas mal d'adjectifs en -teur, etc. auxquels ne correspond pas un nom d'action en -tion, tels :

(7) (a) accompagnateur, amateur, armateur, assesseur, blasphémateur, calculateur, calomniateur, censeur, commentateur

(b) adjectif, alternatif, comparatif, consécutif

(c) aratoire, blasphématoire, combinatoire, dédicatoire

(ii) On rencontre non seulement des adjectifs en-teur, etc. dont le sens correspond à celui du nom d'action plutôt qu'à celui du verbe, mais aussi le cas contraire. Qu'on compare les exemples de (8), où 1 et 2 indiquent deux sens différents distingués dans Dubois e.a. (1966) :
(8) (a) adhérer 1-2
(b) expédier $1-2$
adhésion 2
(c) fixer $1-2$
expédition 1
fixation 1
adhésif 1
expéditeur 1
fixateur 2
tentation 2
tentateur 2
expéditif 2
fixatif 2
tentative 1

(iii) On constate que dans l'histoire du français certains adjectifs en -teur etc. ont existé avant le nom d'action correspondant en -tion. Comparez (9), dont les données sont empruntées à Bloch et von Wartburg $(1960)$ :
(9) (a) administrateur (vers 1180)
(b) agresseur (1327)
(c) auditeur (1200)
(d) collecteur (1330)
(e) créateur (12)
(f) déprédateur (vers 1285)

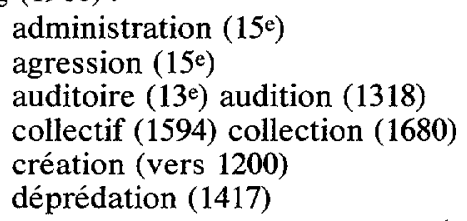

Nous ne tiendrons pas compte des arguments touchant les mots en -tif et toire puisqu'ils ne concernent pas notre analyse. Nous allons répondre tour à tour aux trois points de Zwanenburg en ce qui a trait aux mots en -teur.

(i) Qu'on trouve pas mal d'adjectifs (nous voulons lire "adjectifs et noms") auxquels ne correspond pas un nom d'action, n'est pas tout à fait ce que nous avons constaté. Zwanenburg semble présenter ses exemples comme «quelquesuns parmi tant d'autres »; il en donne 9 pour les -teur; la liste n'est pas complète mais elle représente la moitié dans l'ordre alphabétique car pour la compléter nous devons ajouter: cultivateur, dessinateur, dispensateur, lecteur, ordonnateur, sauveteur, scrutateur, serviteur, triomphateur et le paradigme Xculteur. Retranchons de la liste de Zwanenburg deux mots qui n'ont pas leur place: assesseur et censeur (ce ne sont pas des mots en -teur). Nous avons en tout 17 mots et le paradigme Xculteur. Vu le nombre de formes en -teur que nous avons vu dériver des -tion, à notre sens une vingtaine de contre-exemples (que nous avons déjà traités dans la partie précédente) n'inflrment pas à eux seuls notre hypothèse.

(ii) L'argument à partir des différences de sens est faible. Il n'y a qu'un seul contre-exemple qu'on puisse retenir : fixationifixateur.

(iii) Des six contre-exemples présentés, enlevons (b), agresseur, qui n'est pas un mot en -teur. Il en reste cinq. Mais même s'il y en avait plus, qu'est-ce que 
cela aurait changé? Nous ne faisons qu'expliquer une réalité synchronique du français qui est le fait que les locuteurs contemporains dérivent les mots en -teur des mots en -tion, peu importent pour eux les données diachroniques.

En bref, les contre-exemples de Zwanenburg n'ont pas à notre sens suffisamment de poids pour infirmer le traitement que nous avons proposé pour les X-t-eur. Ces contre-exemples ne constituent, comme nous l'avons déjà dit, qu'un nombre infime si on les compare à la masse de paires régulières. En outre, le fait que ces contre-exemples existent est secondaire pour notre analyse, bien que pertinent, car tout ce qu'ils permettent de dire c'est que les locuteurs ont fait l'analyse «X-t-ion dérive de $X$-t-eur» sur la foi de centaines de paires régulières et en dépit de ce petit nombre d'exceptions. En fait, notre analyse est confirmée par la coexistence d'un nombre assez considérable de paires du type programmeur programmateur, preuve flagrante de l'existence des deux bases : $\mathrm{Xv}$ et $\mathrm{X}$-t-ionN, fait dont l'étude de Zwanenburg ne tient pas compte.

$\mathrm{L}$ 'analyse que nous proposons est fondée sur l'existence des X-t-ion correspondant aux X-t-eur, (nous n'avons pas à postuler de base fictive comme Aronoff). Il est compréhensible que Zwanenburg, qui cherche à évacuer les règles de troncation, tente de réfuter toute analyse qui fait des mots en -tion les bases des mots en -teur; car s'il admettait une telle analyse il serait obligé de convenir que la solution la plus simple et la plus conforme à l'intuition des locuteurs est justement la troncation de -ion, alors que son modèle écarte les règles de troncation. La seule solution qui s'offrirait à Zwanenburg, s'il acceptait de rendre compte des mêmes faits que nous avons observés, tout en renonçant aux règles de troncation, serait de marquer les radicaux dérivés (liés) de type Xat (tel administrat-) d'un trait [+eur]; car dans sa liste de radicaux dérivés il n'aurait pas d'autre manière de reconnaître ceux prenant -eur, comme administrat (que l'on retrouve dans administrat-ion) et ceux qui ne servent pas de base à -eur tel comparat- (que l'on retrouve dans comparat-if mais non dans un mot $\mathrm{X}$-t-ion). Nous avons déjà indiqué qu'une telle solution, consistant à marquer les radicaux au lexique, est peu compatible avec le caractère productif de la dérivation des formes X-t-eur.

\subsection{Mécanisme de la dérivation des $X$-t-eur à partir des $X$-t-ion}

Nous croyons avoir démontré que la seule analyse qui convienne pour les mots en -teur du français est celle qui les fait dériver directement des noms en tion correspondants; cette analyse reflète une réalité de la dérivation française car, comme le faisait remarquer Goosse (cf. 2.2) nombreux sont les mots qui doivent leur création à ce que cet auteur appelle une substitution de suffixes. Mais, comme nous l'avons déjà mentionné, selon nos observations personnelles, la dérivation française s'opère également par «substitution» de préfixes comme en témoignent les mots suivants extraits des néologismes de Dubois (1962): adsorption et désorption (d'absorption); décélération (d'accélération); déflation (d'inflation); déflexion (de réflexion); déglutination (d'agglutination); extrusion (d'intrusion); etc. On remarquera que ces mots n'ont pas de corrélat verbal, et n'ont pu que dériver du mot en -tion. correspondant. 
Examinons maintenant les sept exemples ci-dessous, qui illustrent les sept groupes de mots constituant la classe X-t-eur. Ces mots sont suivis de la X-t-ion ayant servi de base à leur dérivation :

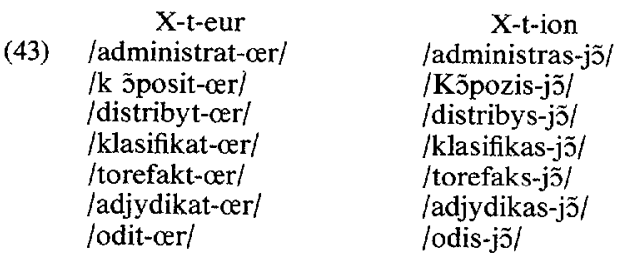

Il semble que nous ayons affaire à une permutation entre $/ j \mathfrak{j} /$ et $/ œ r /$. Néanmoins, /œr/ est toujours précédé de / $\mathrm{t} /$ alors que $/ j \tilde{j} /$ est précédé de $/ \mathrm{s} /$. Il faut donc tenir compte de ce changement morphophonologique. Cette règle $|s| \rightarrow|t|$ n'a rien d'ad hoc si l'on considère l'alternance qui existe souvent en français, au sein d'un même radical, entre ces deux consonnes :

(a)

$\quad$ /t/
aseptique
autoptique
écliptique
elliptique
Haîti
mentir
partie
prophète
sentir
synoptique

$\quad$ /s/
asepsie
autopsie
éclipse
ellipse
Haïtien
mensonge
partiel
prophétie
sens
synopsis

(On remarquera par les quelques exemples ci-dessous que c'est souvent le contexte $+\mathrm{ja}$ qui déclenche la conversion $\mathrm{du} / \mathrm{s} /$ en $/ \mathrm{t} /$.)

(b)

attentif correctif
préventif supplétif

(c) diffamatoire directoire éliminatoire

(d) acte ondulatoire dictée fracture secteur

Voyons maintenant comment Aronoff pourrait intégrer dans son modèle cette analyse qui semble la plus simple pour décrire les faits observés. Nous devons dire d'emblée que notre analyse peut s'inscrire techniquement dans le modèle d'Aronoff qui a les mécanismes pour en rendre compte, mais que théoriquement elle cause, une fois de plus, un problème, puisqu'elle viole le principe de la base unitaire comme le faisaient déjà les Xv-eur (cf 1.3). Nous pensons qu'Aronoff devrait reconsidérer le principe de la base unitaire; s'il s'applique peut-être pour l'anglais, il est fort douteux qu'il puisse constituer un principe universel : en effet, plusieurs études ont démontré qu'un même affixe pouvait s'adjoindre productivement à des bases de catégories différentes (ex.: Drapeau et Boulanger (1982), pour le suffixe -erie en français du Québec); un cas patent pour la réfutation de ce principe semble être celui du montagnais : 
Lynn Drapeau (1980) a montré d'une façon très convaincante que la contrainte métathéorique que constitue l'hypothèse de la formation d'un mot à partir d'un autre déjà existant et membre d'une catégorie lexicale majeure (hypothèse à la base de la théorie d'Aronoff) ne fait pas partie de la grammaire universelle; l'analyse de Lynn Drapeau se fonde sur la formation extrêmement productive de mots en montagnais à partir de racines qui n'apparaissent jamais comme morphèmes libres. Si ce principe doit être réfuté, ce qui semble être le cas, il faut par voie de conséquence réfuter celui de la base unitaire puisque les racines, auxquelles ces affixes s'adjoignent très productivement, ne peuvent être classées en termes de catégories lexicales majeures.

Donc, nous supposerons qu'Aronoff (1976) (modèle révisé pour ne pas tenir compte du principe de la base unitaire) rendrait compte de la dérivation en teur à partir des mots en -tion à l'aide de trois règles; une règle de formation de mot qui adjoint -eur à la base nominale suffixée en -ion, une règle de troncation qui efface le suffixe -tion et une règle d'allomorphie qui convertit le /s/ en $/ \mathrm{t} /$. Voici les trois règles formalisées ci-dessous, la première formant avec la règle (8) le schéma de règles (45) :

(45) 1. Règle de formation de mots

a. $\quad[\mathrm{X}] \leftrightharpoons \rightarrow[\mathrm{X}]$ v eur $] \mathrm{N}$

Fonction sémantique de la règle

«fait l'action de $X »$ où $X=V$

«fait quelque chose avec $X$ » où $X=N$

b. Formes de la base :

1. XV (ex.: donne)

2. X-t-ion (ex.: indication)

Condition: $X$ ne doit pas être un radical de type finir

3. $\mathrm{XN}_{\mathrm{N}}$ (ex. : ferraille)

2. Règle de troncation

$[\mathrm{X}+$ ion $] \mathrm{N}+$ eur

$12 \quad 3 \rightarrow 103$

3. Règle d'allomorphie $s \rightarrow t$

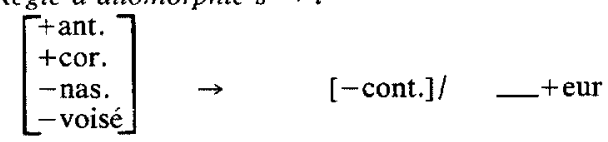

Note: Cette même règle d'allomorphie s'applique devant -if, -oire, etc.

Après la révision proposée, nous constatons que le modèle permet de rendre compte simplement et efficacement de la formation des X-t-eur. Nous allons maintenant essayer de voir si notre analyse peut s'intégrer dans un modèle à la Lieber. Nous avons vu auparavant que Lieber (1980) aurait eu de sérieux problèmes à rendre compte des X-t-eur à partir des radicaux verbaux dérivés du genre $\mathrm{Xat}$ (ex.: administr-at-) où $\mathrm{X}=$ radical verbal simple et libre. Exactement comme nous l'expliquions pour Zwanenburg (voir fin de 3.1), elle aurait été obligée de marquer chaque radical dérivé d'un trait [ + eur] étant donné qu'elle n'aurait pas eu le moyen de stipuler plus simplement que l'existence de ces formes est liée à celle des X-t-ion.

Nous voulons à ce point faire état de l'analyse des formes en -ive (etc.) de l'anglais par Lieber qui se veut être, comme celle de Zwanenburg (1980), une réanalyse des données d'Aronoff. Nous savons qu'Aronoff proposait de passer par la forme en -tion pour dériver les formes en -ive entre autres (cf. 3.1); 
quant à Lieber, elle a recours à l'allomorphie des radicaux pour tous les cas, même là où Aranoff postule des règles d'allomorphie du suffixe. Son modèle lui permet d'entrer au lexique par exemple à la fois conduce et conduct, classe qui sera définie par les règles morpholexicales Xduce $\sim$; grâce à la sous-catégorisation, elle peut stipuler que c'est à conduct que s'adjoindront -ion et -ive. Pour dériver formationiformative, elle entre au lexique form et formate (classe $\mathrm{X} \sim \mathrm{Xate)}$, bien que formate ne soit pas un radical libre; son modèle lui permet d'éviter l'allomorphie du suffixe en -ation qu'Aronoff est obligé de postuler.

Mais Lieber, dont l'analyse est comparable à celle de Zwanenburg, ne propose pas à proprement parler un traitement qui remplace celui d'Aronoff, car elle n'explique d'aucune manière que ces formes en -ive (etc.) sont issues des formes en -tion, si toutefois elles le sont et, partant, elle ne rend pas compte de la productivité du processus. Dans notre cas, ce type d'analyse, nous l'avons vu, ne convient pas car nous voulons expliciter la relation directe qui existe entre $\mathrm{X}$-t-eur et $\mathrm{X}$-t-ion; étant donné que Lieber ne prévoit pas de règle de troncation, elle n'a donc aucun moyen de rendre compte de la productivité de la règle en partant du radical nominal. Notre analyse semble donc s'inscrire mieux, jusqu'ici, dans un modèle à la Aronoff que dans un cadre à la Lieber. Nous voulons cependant proposer une autre solution qui à notre avis rend mieux compte d'un facteur important: l'intuition des locuteurs. En effet les locuteurs $\mathrm{du}$ français dérivent les mots en -teur des mots en -tion, mais certaines «irrégularités» de la dérivation qui peuvent être révélées par des formations du type combinateur, mot dérivé probablement de combinatoire, puisque nous n'avons pas *combination nous incitent à penser qu'en fait les locuteurs ne forment directement leur mot en -teur sur le mot en -tion que dans la mesure où ils essaient d'extraire la base du mot en -tion pour dériver le mot en -teur. Cela signifie que ce que nous avons appelé jusque là, comme Goosse (1975), une substitution de suffixe serait en quelque sorte un processus se déroulant en deux temps: dans un premier temps, une règle de formation de radical vient tronquer le suffixe en place; dans un deuxième temps, une règle de formation de mot vient adjoindre le suffixe. Faisant cette hypothèse, nous proposons donc les deux règles, suivies d'une règle d'allomorphie, formalisées de la façon suivante:

(46) 1. Règle de formation de radical

[X - ion]

$1 \quad 2 \rightarrow 1 \varnothing$

2. Règle de formation de mot

$[\mathrm{X}] \rightarrow[[\mathrm{X}]$ eur $] \mathrm{N}$

où $\mathrm{X}=\mathrm{V}, \mathrm{N}$ ou un radical lié formé par la règle précédente

3. Règle d'allomorphie $x \rightarrow t$

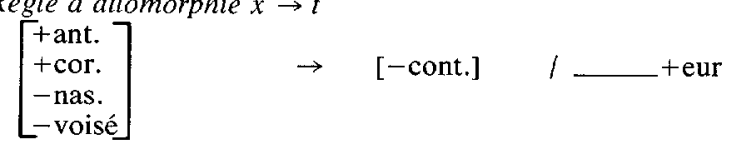

Il n'est plus question d'inscrire maintenant cette solution dans le modèle d'Aronoff pour la simple raison que ce modèle est basé sur l'hypothèse de la dérivation d'un mot à partir d'un autre déjà existant. C'est ce principe qui oblige Aronoff à ordonner ses règles ainsi : 
1. règle de formation (opérant sur un mot existant),

2 . règle de troncation et 3 . règle d'allomorphie.

Dans notre cas, notre règle de formation de mots peut opérer sur un morphème lié et sur ce point nous rejoignons Lieber (1980).

\section{CONCLUSIONS GÉNÉRALES}

Nous croyons avoir montré que toutes les formes en -eur ne peuvent être analysées de la même manière : il faut d'emblée faire une distinction entre celles qui finissent en -eur et celles qui finissent en -teur, où $/ t /$ ne fait pas a priori partie du radical. Nous avons vu que même les premières ne peuvent être toutes décrites comme déverbales (la majorité cependant le peuvent) : une certaine quantité d'entre elles dérivent d'une base nominale monomorphémique.

Nous avons vu que les formes en -teur ne peuvent être considérées comme déverbales en français contemporain mais comme dérivées d'une forme en -tion. Nous avons présenté deux possibilités pour la dérivation de ces mots en -teur: soit une solution à la Aronoff en ayant recours à la suffixation de -eur au mot en tion, à la troncation de -ion et à la conversion de $/ \mathrm{s} / \mathrm{en} / \mathrm{t} / \mathrm{devant}-\mathrm{eur}$. Soit postuler une règle de formation de radical, qui tronque le suffixe-tion du mot en tion, suivie de la suffixation de -eur et de la règle morphophonologique /s/ $\rightarrow$ $/ \mathrm{t} / / \ldots+$ +eur. Cette dernière solution, plus conforme à l'intuition des locuteurs que celle que le modèle d'Aronoff nous impose, pourrait très bien s'intégrer dans un modèle à la Lieber pourvu qu'il prévoie des règles de formation de radicaux, qui sont indépendamment motivées pour le français comme nous pouvons le voir par les quelques dérivés de mots en -tion ci-dessous (certains ont déjà été cités) :

(47) décélêration, d'accélération (troncation du préfixe en place et ajout du nouveau préfixe) déflation, d'inflation (id.) déglutination, d'agglutination (id.)

déplantation, d'implantation (id.) détoxication, d'intoxication (id.)

Lieber (1980), dans l'état où est son modèle, ne peut prévoir la formation de nouveaux radicaux et du fait même ne peut rendre d'un processus dérivationnel assez productif pour le français. Par exemple, étant donné que son lexique ne contient que les radicaux déjà existants dans les dérivés, Lieber ne peut dériver décélération puisqu'elle n'a pas accès à -célération qui n'existe dans aucun dérivé mais qui a été formé par troncation de ac- de accélération.

Ces règles de formation de radicaux, ainsi que des règles de réajustement, devraient faire partie du modèle de Lieber si ce modèle veut s'appliquer au français.

N.B. : Références : Meta, vol. 27, n 3, p. 330.

Antoine Di-Lillo 